\title{
Photon-jet events as a probe of axionlike particles at the LHC
}

\author{
Daohan Wang, ${ }^{1,2,3,+}$ Lei Wu $\odot,{ }^{1, *}$ Jin Min Yang $\oplus^{2,3, \S}$ and Mengchao Zhang $\oplus^{4, \dagger}$ \\ ${ }^{1}$ Department of Physics and Institute of Theoretical Physics, Nanjing Normal University, \\ Nanjing 210023, China \\ ${ }^{2}$ CAS Key Laboratory of Theoretical Physics, Institute of Theoretical Physics, \\ Chinese Academy of Sciences, Beijing 100190, China \\ ${ }^{3}$ School of Physical Sciences, University of Chinese Academy of Sciences, Beijing 100049, China \\ ${ }^{4}$ Department of Physics and Siyuan Laboratory, Jinan University, \\ Guangzhou 510632, People's Republic of China
}

(Received 9 February 2021; accepted 25 October 2021; published 17 November 2021)

\begin{abstract}
Axionlike particles (ALPs) are predicted by many extensions of the Standard Model. When ALP mass lies in the range of $\mathrm{MeV}-\mathrm{GeV}$, the cosmology and astrophysics will be largely irrelevant. In this work, we investigate such light ALPs through the ALP-strahlung process $p p \rightarrow V a(\rightarrow \gamma \gamma)$ at the $14 \mathrm{TeV}$ LHC with an integrated luminosity of $3000 \mathrm{fb}^{-1}$ (high-luminosity LHC). With the photon-jet algorithm, we demonstrate that our approach can probe the mass range of ALPs, which has been inaccessible in previous LHC experiments. The obtained result can surpass the existing limits on ALP-photon coupling in the ALP mass range from 0.3 to $10 \mathrm{GeV}$.
\end{abstract}

DOI: 10.1103/PhysRevD.104.095016

\section{INTRODUCTION}

Searching for new particles is one of the crucial tasks in the LHC experiment. Light pseudo-scalars, such as axionlike particles (ALPs), are theoretically well motivated. They generically appear in models with the spontaneous breaking of a global symmetry [1-4] or in the compactifications of string theory [5-7]. Additionally, ALPs may have connections with electroweak phase transition [8] and play a key role in solving the hierarchy problem [9].

In general, the ALP masses and couplings to Standard Model (SM) particles are independent parameters. When ALP masses are below the MeV scale, they are already subject to many constraints from cosmological and astrophysical observations [10,11]. Additionally, such light ALPs can serve as cold dark matter [12-14] and be explored by various astrophysical and terrestrial anomalies [15-18]. While for ALPs in the mass range of $\mathrm{MeV}-\mathrm{GeV}$, the above cosmological and astrophysical bounds will vanish. However, ALPs can have sizable contributions to low-energy

\footnotetext{
*Corresponding author. leiwu@njnu.edu.cn

Corresponding author. mczhang@jnu.edu.cn

*wangdaohan@itp.ac.cn

§jmyang@itp.ac.cn

Published by the American Physical Society under the terms of the Creative Commons Attribution 4.0 International license. Further distribution of this work must maintain attribution to the author(s) and the published article's title, journal citation, and DOI. Funded by SCOAP ${ }^{3}$.
}

observables in particle physics. Recently, many works have been devoted to searching for ALPs in intensity frontiers [19-23].

On the other hand, the ALPs can be directly produced at high-energy colliders [24-32]. A considerable region of parameter space of ALPs has been constrained by the Large Electron-Positron Collider (LEP) and LHC data. For instance, ALPs can be searched for from the processes $e^{+} e^{-} \rightarrow \gamma a(\rightarrow \gamma \gamma)$ and $Z \rightarrow a \gamma$ at LEP [33]. The nonresonant production process of ALPs, such as $p p \rightarrow Z Z$, has been proposed at the LHC [34]. The rare decays of Higgs boson $h \rightarrow Z a(\rightarrow \gamma \gamma)$ and $h \rightarrow a(\rightarrow \gamma \gamma) a(\rightarrow \gamma \gamma)$ have been proposed to probe the ALP coupling to the photon in terms of the ALP mass on a future complete LHC run-3 dataset in the future [35].

However, current LHC analyses are sensitive to the ALPs in the parameter region $m_{a} \gtrsim 10 \mathrm{GeV}$. When ALPs become light, they can be highly boosted, and thus the two photons from the ALP decay are recognized as one object in the detector. This will lead to an interesting signature "photon jet" at the LHC. In this work, we will use jet substructure variables to analyze these photon-jet events from the decay of ALPs, as the method proposed in Refs. [36,37]. We will focus on ALPs that only couple to the electroweak vector bosons with a mass in the MeV$\mathrm{GeV}$ range. Such light electroweak ALPs have obtained an increasing amount of interest due to current collider data. In this work, there is no ALP-gluon coupling so that the dominant production channel of electroweak ALPs at the LHC is the ALP-strahlung process $p p \rightarrow V a$, where $V=W, Z$ (see Fig. 1). We find that our proposal can 


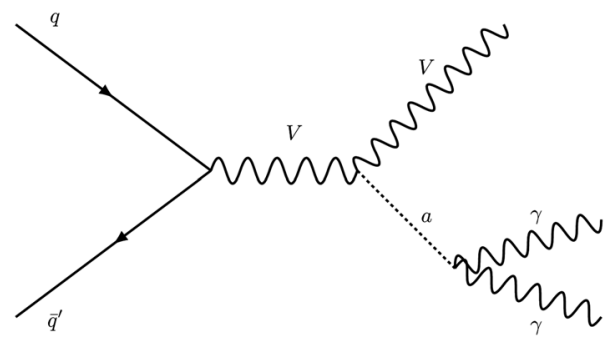

FIG. 1. Feynman diagrams of ALP-strahlung process $p p \rightarrow$ $V a(\rightarrow \gamma \gamma)$ at the LHC, where $V=W, Z$.

extend the LHC sensitivity to parameter space unreachable in previous studies.

\section{MODEL AND PHOTON JET}

The relevant effective interactions of ALP with electroweak gauge bosons up to dimension five is given by [28]

$$
\begin{aligned}
\mathcal{L}_{\text {eff }} \supseteq & \frac{1}{2}\left(\partial^{\mu} a\right)\left(\partial_{\mu} a\right)-\frac{1}{2} m_{a}^{2} a^{2}-C_{B B} \frac{a}{f_{a}} B_{\mu \nu} \tilde{B}^{\mu \nu} \\
& -C_{W W} \frac{a}{f_{a}} W_{\mu \nu}^{i} \tilde{W}^{\mu \nu, i},
\end{aligned}
$$

where the ALP field is denoted by $a$, and the field strengths for the SM gauge groups are denoted as $V_{\mu \nu} \equiv \partial_{\mu} V_{\nu}-\partial_{\nu} V_{\mu}$ and $\tilde{V}_{\mu \nu} \equiv \epsilon_{\mu \nu \rho \sigma} V^{\rho \sigma}$. The $W_{\mu \nu}$ and $B_{\mu \nu}$ are field strengths for $S U(2)_{L}$ and $U(1)_{Y}$, respectively. Both $C_{W W}$ and $C_{B B}$ contribute to the interaction of the ALP with two photons. The dimensionful coupling $g_{a \gamma \gamma}, g_{a W W}, g_{a Z Z}$, and $g_{a \gamma Z}$ are given by

$$
\begin{aligned}
g_{a \gamma \gamma} & =\frac{4}{f_{a}}\left(C_{B B} \cos \theta_{W}^{2}+C_{W W} \sin \theta_{W}^{2}\right), \\
g_{a W W} & =\frac{4}{f_{a}} C_{W W}, \\
g_{a Z Z} & =\frac{4}{f_{a}}\left(C_{B B} \sin \theta_{W}^{2}+C_{W W} \cos \theta_{W}^{2}\right), \\
g_{a \gamma Z} & =\frac{8}{f_{a}} \sin \theta_{W} \cos \theta_{W}\left(C_{W W}-C_{B B}\right),
\end{aligned}
$$

where $\theta_{W}$ is the Weinberg angle. For simplicity, we set $C_{W W}=C_{B B}$ in our study. We consider two ALPstrahlung processes $p p \rightarrow W^{ \pm}\left(\rightarrow \ell^{ \pm} \nu\right) a(\rightarrow \gamma \gamma)$ and $p p \rightarrow$ $Z\left(\rightarrow \ell^{+} \ell^{-}\right) a(\rightarrow \gamma \gamma)$ as our signals. The dominant SM background processes include $V \gamma, V j$, and QCD dijets. In the LHC experiment, when $m_{a} \gtrsim 10 \mathrm{GeV}$, the two photons from ALP decay can be separated enough and identified as $2 \gamma$ events. On the other hand, if ALP mass is lighter than a few hundred $\mathrm{MeV}$, those photons are highly collimated so that they will be detected as single photon events. While between these two mass limits, the two photons will be seen like a photon jet. In this case, we can use jet substructure techniques to discriminate photon jets from single photons and QCD jets [36-41].

We implement the Lagrangian of Eq. (1) in FEYNRULES [42] to generate the corresponding UFO model file. We use MADGRAPH5_AMC@NLO [43] to calculate the production cross sections and generate the signal and background events. Then the parton level events are showered and hadronized by PYTHIA8 [44]. The detector simulation is performed by DELPHES [45]. FASTJET [46] is used for jet clustering. The electron and muon identification efficiencies are taken as default values. Based on the energy-flow algorithm [47], we cluster EflowPhotons, EflowNeutralHadrons, and ChargedHadrons into jets by using the anti- $k_{t}$ algorithm [48] with $R_{j}=0.4$. Only the leading jet with $p_{T}>50 \mathrm{GeV}$ for each event is retained for further analysis. Then we recluster the rest of the energy deposits in each jet using the $k_{t}$ algorithm $[49,50]$, which determines a recombination tree for the jets. In addition, we also consider the pileup interaction effect. The low- $Q^{2}$ soft QCD pileup events are generated by PYTHIA8 and then simulated by DELPHES. We take the default parametrization in the Compact Muon Solenoid (CMS) card to distribute the minimum-bias pileup events and hard scattering events in time and $z$ positions. The average amount of pileup events per bunch crossing is considered to be 40 . In addition, it should be noted that the calibration of the jet energy scale is one of the main uncertainties in the jet analysis at the LHC. Since the traditional photon isolation criterion is not used, the new method of calibrating the photon-jet energy scale based on a full simulation of detector and real data is needed. This is challenging for a phenomenological study and is beyond the scope of our work.

To discriminate the signal events from the SM backgrounds, we use jet substructure algorithm to select our photon-jet events. We generate $p p \rightarrow Z(\rightarrow \nu \bar{\nu}) a$, $p p \rightarrow Z(\rightarrow \nu \bar{\nu}) j$, and $p p \rightarrow Z(\rightarrow \nu \bar{\nu}) \gamma$ as the training samples of photon jet, QCD jet, and single photon events, respectively. According to Refs. [36,37], the following variables are used in our substructure analysis:

(1) The hadronic energy fraction of a jet $\theta_{J}$ is defined as the energy fraction carried by a jet's constituents that belong to the hadronic calorimeter, including the EflowNeutralHadrons and ChargedHadrons in the energy-flow algorithm,

$$
\theta_{J}=\frac{1}{E_{J}}\left(\sum_{i} E_{i}+\sum_{j} E_{j}\right),
$$

where $E_{J}$ is the total energy of the leading jet, and $E_{i}$ and $E_{j}$ are the energy of the $i$ th EflowNeutralHadron and the energy of the $j$ th ChargedHadron, respectively, that are constituents of the jet. Because of isospin symmetry, a QCD jet typically contains about $2 / 3$ charged pions and $1 / 3$ neutral pions, which will decay to a pair of photons. Thus we expect to have a peak at $\theta_{J} \sim 2 / 3\left(\log \theta_{J} \sim-0.2\right)$ for QCD jets. On the other hand, most of the energy of single photons and photon jets are 
deposited in the electromagnetic calorimeter. So the $\log \theta_{J}$ of them are much smaller than that of QCD jets. In Fig. 2, we show the distribution of $\log \theta_{J}$ for QCD jets, single photons, and photon jets in our simulation data.

(2) The number of charged tracks in a jet $N_{\text {track }}$ means the number of charged particles inside a jet. We calculate the angular distance $\Delta R$ between the leading jet and all the tracks with $p_{T}>2 \mathrm{GeV}$. When $\Delta R<R_{j}$, the corresponding track is considered to be inside the leading jet. As mentioned before, a QCD jet typically contains several charged pions, while single photons and photon jets leave no tracks in the tracker. Therefore, the number of tracks associated with QCD jets varies over a broad range; however, the single photon and photon-jet samples are dominated by jets without associated tracks. In Fig. 2, we show the distribution of $N_{\text {track }}$ per jet for QCD-jet, single photon, and photon-jet samples. It can be seen that the variable $N_{\text {track }}$ has a good discrimination power of QCD jets and single photons/photon jets, but cannot distinguish the single photons from photon jets.

(3) $N$-subjettiness is a measure of the number of energetic subjets inside a jet [51,52]. Given a set of $\mathrm{N}$ axes, we can define

$$
\tau_{N}=\frac{\sum_{k} p_{T_{k}} \times \min \left\{\Delta R_{1, k}, \ldots, \Delta R_{N, k}\right\}}{\sum_{k} p_{T_{k}} \times R_{j}},
$$

where $k$ runs over all the constituents of the jet. $p_{T_{k}}$ is the transverse momentum of the $k$ th constituent, and $\Delta R_{l, k}$ is the angular distance between the $l$ th subjet and the $k$ th constituent of the jet. When $N=1, N$-subjettiness describes the energy distribution of the jet. In Fig. 3, it can be seen that the single photon and photon-jet samples peak at lower values of $\log \tau_{1}$, as a comparison with the QCD jets. Additionally, $\tau_{N}$ will decrease rapidly as $N$ is increasing. Thus, the ratio of two $N$-subjettiness $\tau_{a} / \tau_{b}$ can be used to separate the signal and backgrounds. From Fig. 3, we can see that the photon-jet and single photon events have smaller values of $\tau_{2} / \tau_{1}, \tau_{3} / \tau_{1}$, and $\tau_{3} / \tau_{2}$ than the QCD jets.

(4) Functions of energy and $p_{T}$ of subjets are also used. We use jet filtering [53] in FASTJET to recluster the leading jet's constituents using the $k_{T}$ algorithm. After reclustering, we obtain $N$ exclusive $k_{T}$ subjets. We set $N=5$ and take the three hardest subjets to construct two jet substructure variables. One is the fraction of the jet $p_{T}$ carried by the leading subjet $\lambda_{J}$,

$$
\lambda_{J}=\log \left(1-\frac{p_{T_{L}}}{p_{T_{J}}}\right)
$$

and the other is the energy correlation function of the three hardest subjets $\epsilon_{J}$,

$$
\epsilon_{J}=\frac{1}{E_{J}^{2}} \sum_{(i>j) \in N_{\text {hard }}} E_{i} E_{j}
$$

where $p_{T_{L}}$ and $E_{J}$ are the total momentum and energy of a given jet, respectively. $E_{i}$ is the energy of the $i$ th subjet. For the single photon and photon jet, the leading subjet carries most of the energy. Therefore, their distributions of $\lambda_{J}$ have smaller values than QCD jets. As for $\epsilon_{J}$, among the three hardest jets, $\epsilon_{J}$ increases with the number of subjets with similar energy. The photon jet and QCD jets have larger values of $\epsilon_{J}$ than the single photon. Figure 4 presents the corresponding distributions of $\epsilon_{J}$ and $\lambda_{J}$ for signal and backgrounds.

In the following, we implement the above variables in a boosted decision tree (BDT) [54] to enhance the ability of distinguishing signal from background, as in Refs. [36,37]. In practice, we use the Toolkit for Multivariate Analysis [55] package and the "BDTD" option to book BDTs. We use a 200 trees ensemble that requires minimum training events in each node of $2.5 \%$ and a maximum tree depth of 3. Other variables are set at their default values.

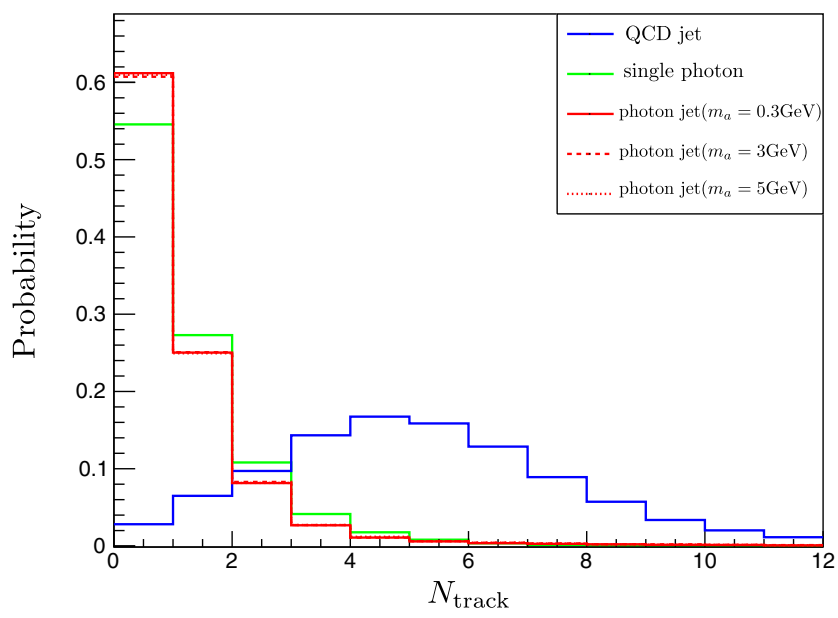

FIG. 2. Distributions of jet substructure variables $\left(\log \theta_{J}, N_{\text {track }}\right)$ for single photon, photon-jet, and QCD jet events. 

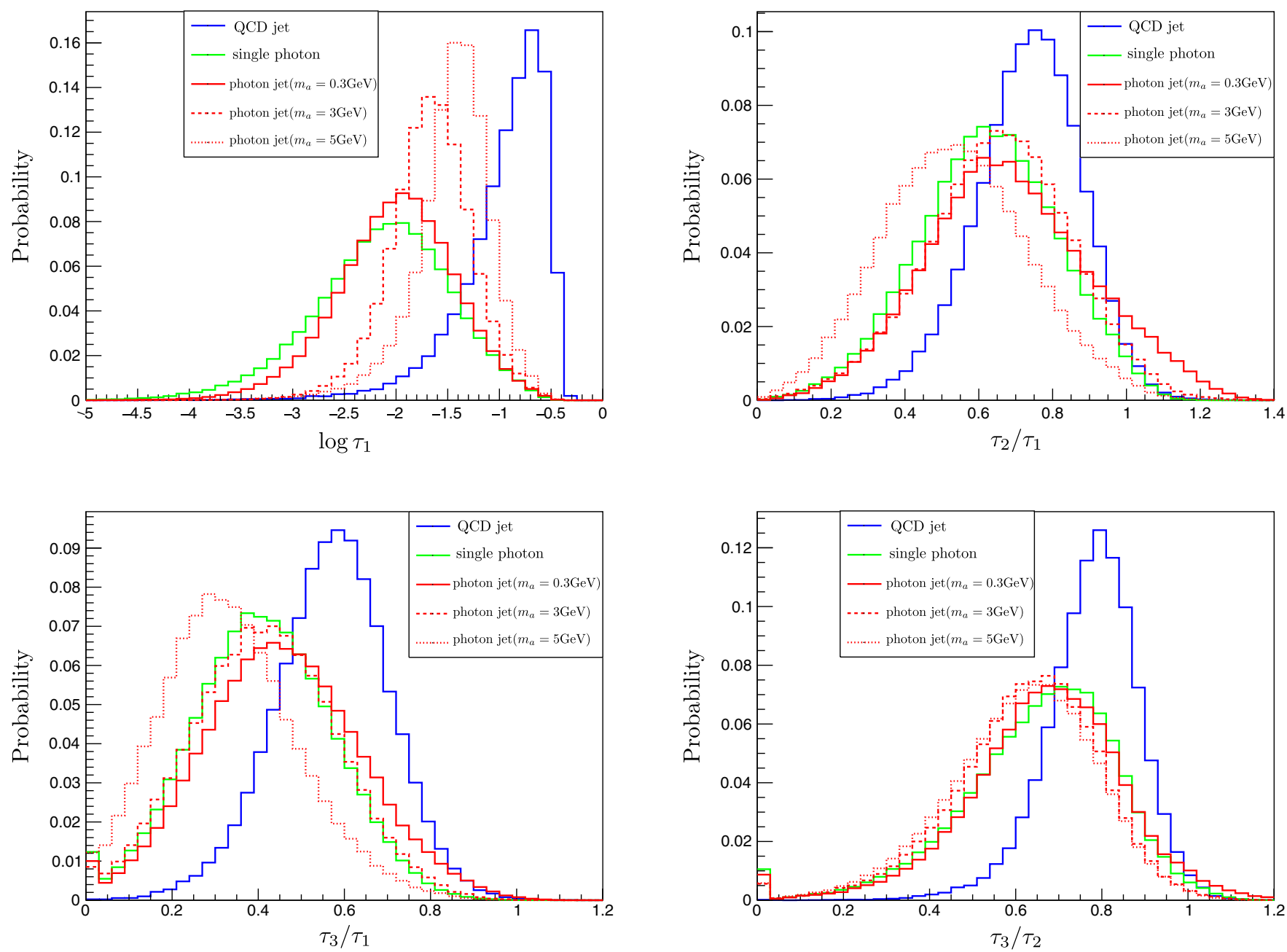

FIG. 3. Distributions of $N$-subjettiness variables $\left(\log \tau_{1}, \tau_{2} / \tau_{1}, \tau_{3} / \tau_{1}, \tau_{3} / \tau_{2}\right)$ for single photon, photon-jet, and QCD jet events.

It is trained using the half of the signal and background events and is tested on the rest of the events. We also demand the Kolmogorov-Smirnov test of the BDT analysis to be greater than 0.01 to avoid overtraining.

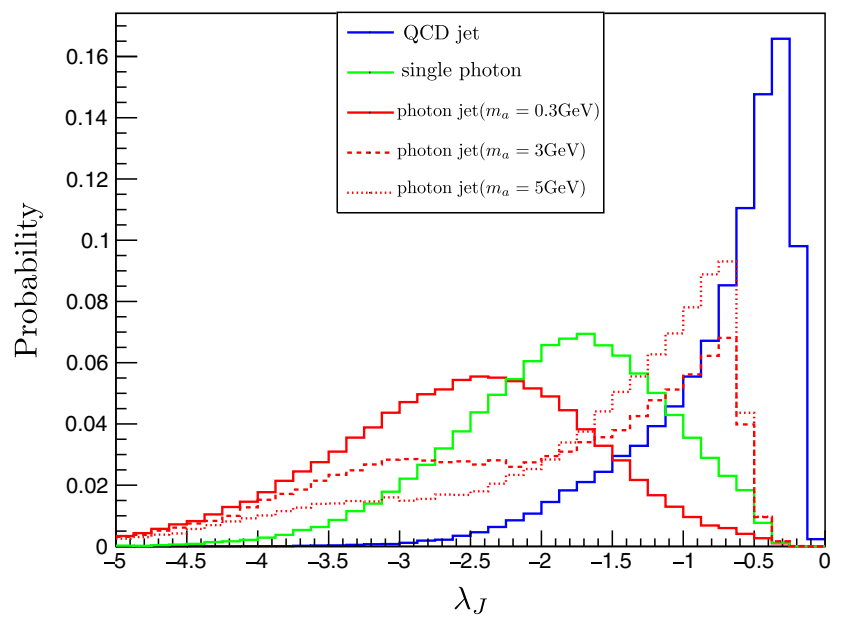

From Fig. 2, we can see that $\theta_{J}$ and $N_{\text {track }}$ offer a good discrimination between photon jets and QCD jets. The other six variables can be used for discrimination between photon jets and single photons. In order to achieve

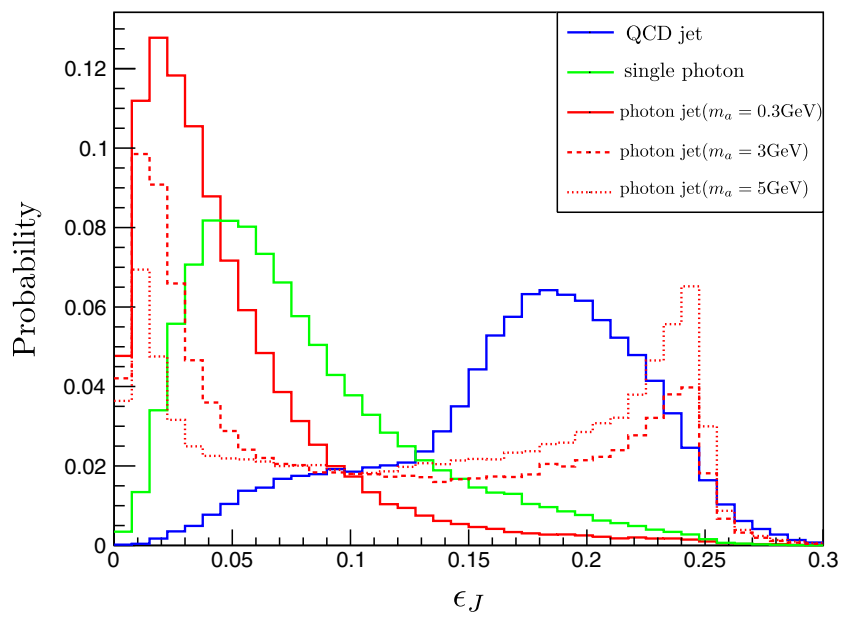

FIG. 4. Distributions of jet substructure variables $\left(\lambda_{J}, \epsilon_{J}\right)$ for single photon, photon-jet, and QCD jet events. 

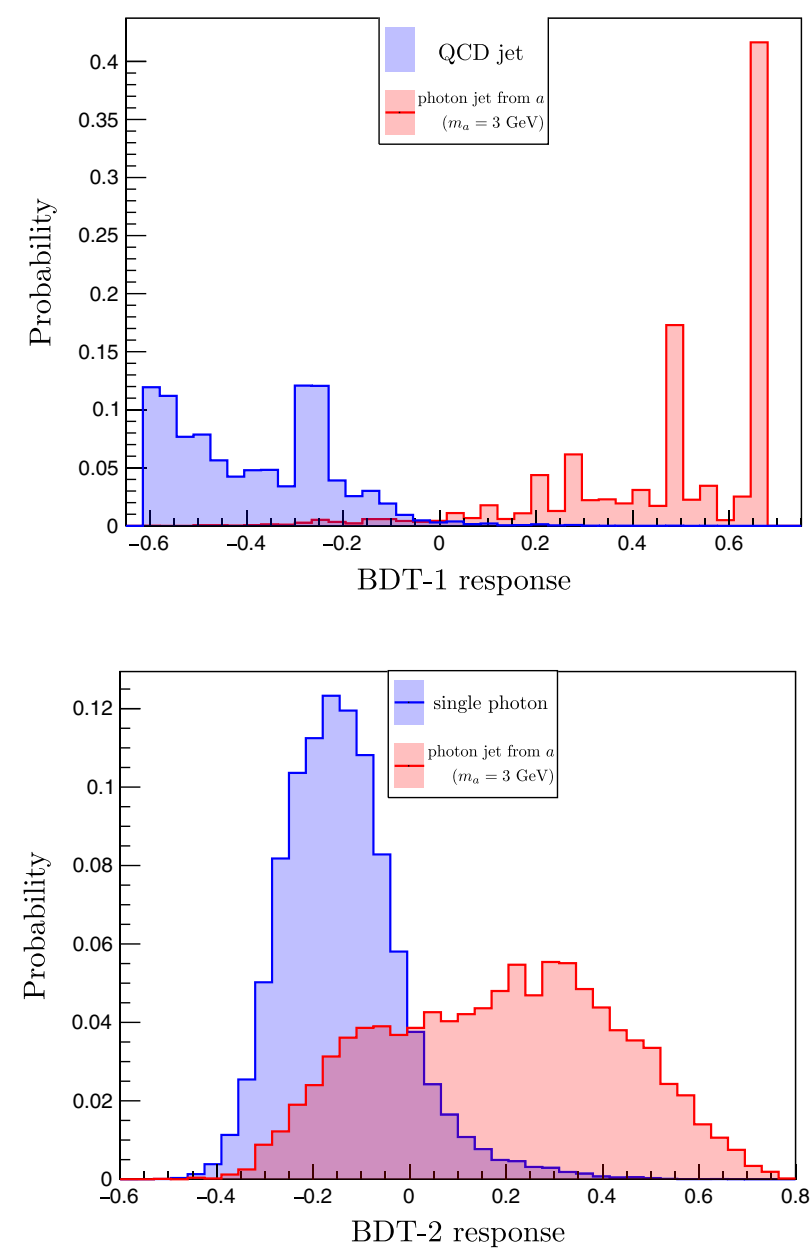

FIG. 5. BDT response distributions of a photon jet as a signal and a single photon and QCD jet as backgrounds, respectively.

simultaneous separation of photon jets, single photons, and QCD jets, we take the $\theta_{J}$ and $N_{\text {track }}$ of photon jets as signal samples and the $\theta_{J}$ and $N_{\text {track }}$ of QCD jets as background samples to train the first BDT, namely, BDT-1. The other six jet substructure variables of photon jets and single photons, as signal samples and background samples, respectively, are used to train the second BDT, namely, BDT-2. In other words, we discriminate photon jets and single photons from QCD jets by using BDT-1, and then discriminate photon jets from single photons by using BDT-2.

After training, BDT can map an event with two sets of variables $\left\{\log \theta_{J}, N_{T}\right\}$ and $\left\{\log \tau_{1}, \tau_{2} / \tau_{1}, \tau_{3} / \tau_{1}, \tau_{3} / \tau_{2}\right.$, $\left.\lambda_{J}, \epsilon_{J}\right\}$ into two BDT responses.
In Fig. 5 we show the BDT responses for $m_{a}=3 \mathrm{GeV}$. In the BDT-1 analysis, photon-jet- and photonlike events tend to get high BDT response, while QCD jetlike events tend to get low response. In BDT-2, a photon-jetlike event tends to get high BDT response, while a photonlike event tends to get a low BDT response. In BDT-1, we identify a jet with BDT response larger than 0 as a photon jet or a single photon, while in BDT-2, we tag a jet with BDT response larger than 0.4 as a photon jet. Since the jet substructure variables are sensitive to the ALP mass, we choose different BDT response cuts for different ALP masses to optimize the search ability.

\section{ALP-STRAHLUNG PROCESS AT THE LHC}

For the ALP-strahlung process $p p \rightarrow W^{ \pm} a$, the final states are characterized by an isolated lepton and one photon jet. We consider the SM backgrounds: QCD dijet, $W^{ \pm} j, W^{ \pm} \gamma, t \bar{t}$, and $t j$. Based on the above analysis, we impose the following cuts to discriminate the signal and backgrounds: (i) exactly one isolated lepton (electron or muon) with $p_{\mathrm{T}}>20 \mathrm{GeV}$ and $|\eta|<2.5$, (ii) the hardest jet with $p_{\mathrm{T}}>50 \mathrm{GeV}$ and $|\eta|<2.5$, and (iii) the hardest jet's BDT-1 response is larger than 0 and BDT-2 response is larger than the corresponding BDT response cut for different ALP masses.

For the ALP-strahlung process $p p \rightarrow Z a$, the final states are characterized by the opposite sign and same flavor charged lepton pair and a photon jet. The main SM backgrounds include $Z \gamma$ and $Z j$. According to our analysis, we discriminate the signal and backgrounds by imposing the following cuts: (i) exactly two leptons with $p_{\mathrm{T}}>$ $20 \mathrm{GeV}$ and $|\eta|<2.5$, (ii) the invariant mass of the oppositely charged lepton pair with same flavor must be within $\left|m_{l l}-m_{Z}\right|<20 \mathrm{GeV}$, (iii) the hardest jet with $p_{\mathrm{T}}>$ $50 \mathrm{GeV}$ and $|\eta|<2.5$, and (iv) the hardest jet's BDT-1 response is larger than 0 and BDT-2 response is larger than the corresponding BDT response cut for each ALP mass.

As an example, we consider a benchmark signal point with $m_{a}=3 \mathrm{GeV}$ and $g_{a \gamma \gamma}=16 \mathrm{TeV}^{-1}$. The cut flows of this benchmark point and the main SM backgrounds are shown in Tables I and II. It can be seen that, after the BDT response cut, all the backgrounds are reduced dramatically. At the end of the cut flow, the largest backgrounds for the processes $p p \rightarrow W^{ \pm} a$ and $p p \rightarrow Z a$ signal are QCD dijet and $Z j$, respectively.

TABLE I. The cut flow of the cross sections (in units of picobarn) of the ALP-strahlung production process $p p \rightarrow a W^{ \pm}$and the corresponding backgrounds at the $14 \mathrm{TeV}$ LHC. The benchmark point is chosen as $m_{a}=3 \mathrm{GeV}$ and $g_{\text {ary }}=16 \mathrm{TeV}^{-1}$.

\begin{tabular}{lcccccr}
\hline \hline Cut flow & Signal & $j j$ & $W^{ \pm} \gamma$ & $W^{ \pm} j$ & $t \bar{t}$ & $t j$ \\
\hline 1 lepton with $p_{\mathrm{T}}>20 \mathrm{GeV}$ and $|\eta|<2.5$ & 36.24 & 19357.15 & 12.31 & 4448.53 & 151.86 & 29.39 \\
The hardest jet with $p_{\mathrm{T}}>50 \mathrm{GeV}$ and $|\eta|<2.5$ & 23.31 & 12893 & 2.54 & 1605.92 & 136.41 & 18.90 \\
The hardest jet's BDT-1 $>0$ and BDT-2 $>0.4$ & 4.14 & 3.30 & 0.001 & 0.77 & 0.094 & 0.012 \\
\hline \hline
\end{tabular}


TABLE II. Same as Table I, but for the ALP-strahlung production process $p p \rightarrow a Z$ and the corresponding backgrounds.

\begin{tabular}{lccc}
\hline \hline Cut flow & Signal & $Z \gamma$ & $Z j$ \\
\hline 2 leptons with $p_{\mathrm{T}}>20 \mathrm{GeV}$ and $|\eta|<2.5$ & 2.65 & 1.93 & 279.90 \\
Oppositely charged lepton pair with same & 2.55 & 1.85 & 275.08 \\
$\quad$ flavorand $\left|m_{l l}-m_{Z}\right|<20 \mathrm{GeV}$ & & 0.44 & 104.476 \\
The hardest jet with $p_{\mathrm{T}}>50 \mathrm{GeV}$ and $|\eta|<2.5$ & 1.79 & 0.0003 & 0.027 \\
The hardest jet's BDT-1 $>0$ and BDT-2 $>0.4$ & 0.29 & & \\
\hline \hline
\end{tabular}

In Fig. 6, we present the $2 \sigma$ bound from our ALPstrahlung production process $p p \rightarrow a W^{ \pm} / Z$ on the plane of the ALP coupling $g_{a \gamma \gamma}$ versus the ALP mass $m_{a}$. We use the Poisson formula $\sqrt{2[(\mathcal{S}+\mathcal{B}) \ln (1+\mathcal{S} / \mathcal{B})-\mathcal{S}]}$ to estimate the sensitivity at the LHC with the luminosity of $3000 \mathrm{fb}^{-1}$, where $\mathcal{S}$ and $\mathcal{B}$ are the number of signal and background events. As the comparison with our results, we also show other relevant bounds on the ALP-photon coupling. Since we only focus on the ALP-electroweak boson couplings in this work, the limits involving the ALP-gluon couplings are not shown in Fig. 6.

It can be seen that our photon-jet method can cover the ALP mass region of $0.3<m_{a}<10 \mathrm{GeV}$ at the LHC, which can extend the current LHC sensitivity of searching for the diphoton resonances in photon fusion and vector boson fusion processes [25,33,60,61]. In addition, our bound on the ALP-photon coupling $g_{a \gamma \gamma}$ is stronger than that derived from the null results of searching for the ALPs in $e^{+} e^{-} \rightarrow 2 \gamma / 3 \gamma$ processes at the LEP [33]. For example,

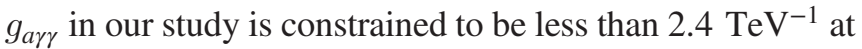

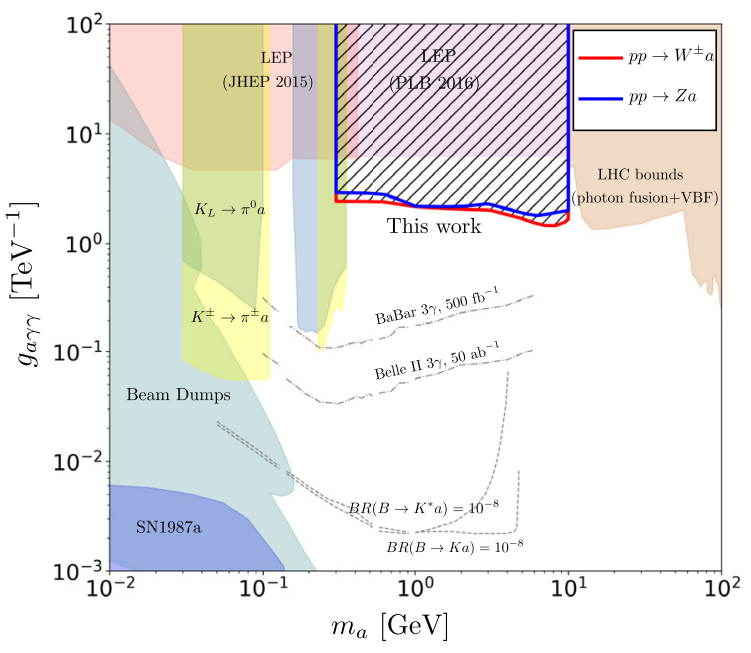

FIG. 6. The $2 \sigma$ exclusion limits on the plane of the ALP mass $m_{a}$ versus the coupling $g_{a \gamma \gamma}$ from the ALP-strahlung production process $p p \rightarrow a W^{ \pm} / Z$ at the LHC with luminosity $3000 \mathrm{fb}^{-1}$. Other limits from the LEP [24,33], SN1987a [56,57], Beam Dump [58,59], ATLAS/CMS [25,33,60,61], and the rare $K$ and $B$ meson decays [19] are also shown. It should be noted that the limits involving the ALP-gluon couplings are not shown but discussed in the context. $m_{a}=0.3 \mathrm{GeV}$ and $1.5 \mathrm{TeV}^{-1}$ at $m_{a}=10 \mathrm{GeV}$. On the other hand, we note that searching for $3 \gamma$ events from the process $e^{+} e^{-} \rightarrow a(\rightarrow \gamma \gamma) \gamma$ and the rare meson decays, such as $K_{L} \rightarrow \pi^{0} a, K^{ \pm} \rightarrow \pi^{ \pm} a$, and $B \rightarrow K^{(*)} a$, in the existing and future low-energy experiments may provide more stringent constraints than ours when $m_{a} \lesssim 5 \mathrm{GeV}$ [19].

In addition, if the ALP-gluon couplings are present and not suppressed by at least 2 orders of magnitude relative to the electroweak ones, the current and future measurements of $B$ meson decays at the $\mathrm{LHCb}$ and $\mathrm{B}$ factories can also give strong exclusion limits for $m_{a}<10 \mathrm{GeV}[62,63]$. However, it should be mentioned that this result is not applicable to our case because the ALP-gluon couplings are absent in our scenario. For the same reason, we do not show the constraints from isolated and energetic photons from hadronic decay of the $Z$ boson at L3 [64] and the searches of ATLAS and CMS for $\gamma \gamma$ resonances in the gluon fusion process [65].

\section{CONCLUSIONS}

In this work we extended the current LHC sensitivity of probing the electroweak ALP via the ALP-strahlung production processes $p p \rightarrow a W^{ \pm} / Z$ in the mass range of $0.3<m_{a}<10 \mathrm{GeV}$ at the $14 \mathrm{TeV}$ high-luminosity LHC (HL-LHC). Since the two photons from ALP decay are not well separated for such a light ALP, we used jet substructure variables and proposed a photon-jet method to discriminate our signal from QCD jets and single photon backgrounds. With the help of the BDT technique, we obtained the $2 \sigma$ bounds on the ALP-photon coupling $g_{a \gamma \gamma}$ as a function of $m_{a}$. The coupling $g_{a \gamma \gamma}>2.4 \mathrm{TeV}^{-1}$ at $m_{a}=0.3 \mathrm{GeV}$ and $g_{a \gamma \gamma}>1.5 \mathrm{TeV}^{-1}$ at $m_{a}=10 \mathrm{GeV}$ can be excluded at $2 \sigma$ level at the 14 TeV HL-LHC. This demonstrates that our approach can not only cover the mass gap $0.3<m_{a}<10 \mathrm{GeV}$ at the LHC, but also surpass the existing LEP bounds. Although the measurements of rare meson decays may also exclude the ALP in the MeVscale mass range, a direct LHC search would provide an independent probe of this parameter space.

\section{ACKNOWLEDGMENTS}

This work is supported by the National Natural Science Foundation of China (NNSFC) under Grants No. 11805161, No. 12075300, No. 11821505, and 
No. 11947118, by Peng-Huan-Wu Theoretical Physics Innovation Center (12047503), by the CAS Center for Excellence in Particle Physics (CCEPP), by the CAS Key Research Program of Frontier Sciences, by a Key R\&D
Program of Ministry of Science and Technology under Grant No. 2017YFA0402204, and by the Key Research Program of the Chinese Academy of Sciences, Grant No. XDPB15.
[1] R. D. Peccei and H. R. Quinn, Phys. Rev. Lett. 38, 1440 (1977).

[2] S. Weinberg, Phys. Rev. Lett. 40, 223 (1978).

[3] F. Wilczek, Phys. Rev. Lett. 40, 279 (1978).

[4] J. E. Kim, Phys. Rev. Lett. 43, 103 (1979).

[5] P. Svrcek and E. Witten, J. High Energy Phys. 06 (2006) 051.

[6] A. Arvanitaki, S. Dimopoulos, S. Dubovsky, N. Kaloper, and J. March-Russell, Phys. Rev. D 81, 123530 (2010).

[7] M. Cicoli, M. Goodsell, and A. Ringwald, J. High Energy Phys. 10 (2012) 146.

[8] G. Ballesteros, J. Redondo, A. Ringwald, and C. Tamarit, Phys. Rev. Lett. 118, 071802 (2017).

[9] P. W. Graham, D. E. Kaplan, and S. Rajendran, Phys. Rev. Lett. 115, 221801 (2015).

[10] G. G. Raffelt, Phys. Rep. 198, 1 (1990).

[11] D. J. E. Marsh, Phys. Rep. 643, 1 (2016).

[12] J. Preskill, M. B. Wise, and F. Wilczek, Phys. Lett. 120B, 127 (1983).

[13] L. F. Abbott and P. Sikivie, Phys. Lett. 120B, 133 (1983).

[14] M. Dine and W. Fischler, Phys. Lett. 120B, 137 (1983).

[15] P. Arias, D. Cadamuro, M. Goodsell, J. Jaeckel, J. Redondo, and A. Ringwald, J. Cosmol. Astropart. Phys. 06 (2012) 013.

[16] J. Jaeckel, J. Redondo, and A. Ringwald, Phys. Rev. D 89, 103511 (2014).

[17] P. Athron et al., J. High Energy Phys. 05 (2021) 159.

[18] C. Gao, J. Liu, L.-T. Wang, X.-P. Wang, W. Xue, and Y.-M. Zhong, Phys. Rev. Lett. 125, 131806 (2020).

[19] E. Izaguirre, T. Lin, and B. Shuve, Phys. Rev. Lett. 118, 111802 (2017).

[20] M. J. Dolan, T. Ferber, C. Hearty, F. Kahlhoefer, and K. Schmidt-Hoberg, J. High Energy Phys. 12 (2017) 094.

[21] M. Bauer, M. Neubert, S. Renner, M. Schnubel, and A. Thamm, Phys. Rev. Lett. 124, 211803 (2020).

[22] D. Banerjee et al. (NA64 Collaboration), Phys. Rev. Lett. 125, 081801 (2020).

[23] Y. Gu, L. Wu, and B. Zhu, arXiv:2105.07232.

[24] K. Mimasu and V. Sanz, J. High Energy Phys. 06 (2015) 173.

[25] S. Knapen, T. Lin, H. K. Lou, and T. Melia, Phys. Rev. Lett. 118, 171801 (2017).

[26] N. D. Barrie, A. Kobakhidze, M. Talia, and L. Wu, Phys. Lett. B 755, 343 (2016).

[27] M. Bauer, M. Neubert, and A. Thamm, J. High Energy Phys. 12 (2017) 044.

[28] I. Brivio, M. B. Gavela, L. Merlo, K. Mimasu, J. M. No, R. del Rey, and V. Sanz, Eur. Phys. J. C 77, 572 (2017).

[29] M. Bauer, M. Heiles, M. Neubert, and A. Thamm, Eur. Phys. J. C 79, 74 (2019).
[30] J. Ebadi, S. Khatibi, and M. Mohammadi Najafabadi, Phys. Rev. D 100, 015016 (2019).

[31] D. Wang, L. Wu, and M. Zhang, Phys. Rev. D 103, 115017 (2021).

[32] J. Ren, D. Wang, L. Wu, J. M. Yang, and M. Zhang, arXiv: 2106.07018 .

[33] J. Jaeckel and M. Spannowsky, Phys. Lett. B 753, 482 (2016).

[34] M. B. Gavela, J. M. No, V. Sanz, and J. F. de Trocóniz, Phys. Rev. Lett. 124, 051802 (2020).

[35] M. Bauer, M. Neubert, and A. Thamm, Phys. Rev. Lett. 119, 031802 (2017).

[36] S. D. Ellis, T. S. Roy, and J. Scholtz, Phys. Rev. Lett. 110, 122003 (2013).

[37] S. D. Ellis, T. S. Roy, and J. Scholtz, Phys. Rev. D 87, 014015 (2013).

[38] B. A. Dobrescu, G. L. Landsberg, and K. T. Matchev, Phys. Rev. D 63, 075003 (2001).

[39] S. Chang, P. J. Fox, and N. Weiner, Phys. Rev. Lett. 98, 111802 (2007).

[40] N. Toro and I. Yavin, Phys. Rev. D 86, 055005 (2012).

[41] P. Draper and D. McKeen, Phys. Rev. D 85, 115023 (2012).

[42] A. Alloul, N. D. Christensen, C. Degrande, C. Duhr, and B. Fuks, Comput. Phys. Commun. 185, 2250 (2014).

[43] J. Alwall, R. Frederix, S. Frixione, V. Hirschi, F. Maltoni, O. Mattelaer, H. S. Shao, T. Stelzer, P. Torrielli, and M. Zaro, J. High Energy Phys. 07 (2014) 079.

[44] T. Sjöstrand, S. Ask, J. R. Christiansen, R. Corke, N. Desai, P. Ilten, S. Mrenna, S. Prestel, C. O. Rasmussen, and P.Z. Skands, Comput. Phys. Commun. 191, 159 (2015).

[45] J. de Favereau, C. Delaere, P. Demin, A. Giammanco, V. Lemaître, A. Mertens, and M. Selvaggi (DELPHES 3 Collaboration), J. High Energy Phys. 02 (2014) 057.

[46] M. Cacciari, G. P. Salam, and G. Soyez, Eur. Phys. J. C 72, 1896 (2012).

[47] CMS Collaboration, Particle-Flow Event Reconstruction in CMS and Performance for Jets, Taus, and MET, Reports No. CMS-PAS-PFT-09-001 and No. CMS-PAS-PFT-09001 (2009).

[48] M. Cacciari, G. P. Salam, and G. Soyez, J. High Energy Phys. 04 (2008) 063.

[49] S. Catani, Y. L. Dokshitzer, M. H. Seymour, and B. R. Webber, Nucl. Phys. B406, 187 (1993).

[50] S. D. Ellis and D. E. Soper, Phys. Rev. D 48, 3160 (1993).

[51] J. Thaler and K. Van Tilburg, J. High Energy Phys. 03 (2011) 015.

[52] J. Thaler and K. Van Tilburg, J. High Energy Phys. 02 (2012) 093. 
[53] J. M. Butterworth, A. R. Davison, M. Rubin, and G. P. Salam, Phys. Rev. Lett. 100, 242001 (2008).

[54] B. P. Roe, H.-J. Yang, J. Zhu, Y. Liu, I. Stancu, and G. McGregor, Nucl. Instrum. Methods Phys. Res., Sect. A 543, 577 (2005).

[55] A. Hocker et al., arXiv:physics/0703039.

[56] A. Payez, C. Evoli, T. Fischer, M. Giannotti, A. Mirizzi, and A. Ringwald, J. Cosmol. Astropart. Phys. 02 (2015) 006.

[57] J. Jaeckel, P. C. Malta, and J. Redondo, Phys. Rev. D 98, 055032 (2018).

[58] B. Döbrich, J. Jaeckel, F. Kahlhoefer, A. Ringwald, and K. Schmidt-Hoberg, J. High Energy Phys. 02 (2016) 018.

[59] B. Döbrich, J. Jaeckel, and T. Spadaro, J. High Energy Phys. 05 (2019) 213; 10 (2020) 046(E).
[60] G. Aad et al. (ATLAS Collaboration), J. High Energy Phys. 01 (2013) 086.

[61] J. Jaeckel, M. Jankowiak, and M. Spannowsky, Phys. Dark Universe 2, 111 (2013).

[62] X. Cid Vidal, A. Mariotti, D. Redigolo, F. Sala, and K. Tobioka, J. High Energy Phys. 01 (2019) 113; 06 (2020) 141(E).

[63] J. P. Lees et al. (BABAR Collaboration), Phys. Rev. Lett. 107, 221803 (2011).

[64] O. Adriani et al. (L3 Collaboration), Phys. Lett. B 292, 472 (1992).

[65] A. Mariotti, D. Redigolo, F. Sala, and K. Tobioka, Phys. Lett. B 783, 13 (2018). 